Proyecciones Journal of Mathematics

Vol. 30, No 3, pp. 369-399, December 2011.

Universidad Católica del Norte

Antofagasta - Chile

\title{
Schauder basis in a locally $K$ - convex space and perfect sequence spaces
}

\author{
R. AMEZIANE \\ UNIVERSITÉ SIDI MOHAMED BEN ABDELLAH, MOROCCO
}

\author{
A. EL AMRANI \\ UNIVERSITÉ SIDI MOHAMED BEN ABDELLAH, MOROCCO \\ and \\ M. BABAHMED \\ UNIVERSITÉ MOULAY ISMAIL, MOROCCO \\ Received : July 2010. Accepted : September 2011
}

\begin{abstract}
In this work, we are dealing with the natural topology in a perfect sequence space and the transfert of topologies of a locally $K-$ convex space $E$ with a Schauder basis $\left(e_{i}\right)_{i}$ to such Space. We are also interested with the compatible topologies on $E$ for which the basis $\left(e_{i}\right)_{i}$ is equicontinuous, and the weak basis problem. Finally, we give some applications to barrelled Spaces and $G$-Spaces.
\end{abstract}

Keywords : non archimedean analysis, locally $K$ - convex spaces, Schauder basis, the weak basis theorem, compatible topologies, perfect sequence spaces, $K$ - barrelled spaces and $G$-spaces.

AMS subject class. (2000): 46S10 


\section{Introduction}

The perfect sequence spaces on a field $K$ have been widely studied by several authors, either in the classical case $(K=\mathbb{R}$ or $K=\mathbb{C})$ Garling [18] and [19], Köthe [23], ..., or in the case of $K$ is a non-archimedean valued field Monna [25], Dorleyn [14], De Grande-De Kimpe [8], ....The importance of the sequence spaces lies on the fact that each space which is locally convex and having a Schauder basis is isomorphic to the sequence space. Thus, instead of studying the spaces that are locally convex and having a Schauder basis one only has to study the sequence spaces.

In this work we are going to establish a way of transforming topologies between this space and a perfect sequence space $\Lambda$, where

$$
\Lambda=\left\{\left(\lambda_{i}\right)_{i} \in \omega: \sum_{i=1}^{\infty} \lambda_{i} e_{i} \text { converges in }(E, \tau)\right\} \text { and }\left(e_{i}\right)_{i} \text { is a Schauder }
$$

basis of a locally $K$-convex space $(E, \tau)$ in question ( $K$ is a non-archimedean valuated field complete with a non trivial valuation ). This study will allow us to solve the following problem:

(1) if $\left(e_{i}\right)_{i}$ is a Schauder basis of a locally $K$ - convex space $(E, \tau)$ $(l K c s)$, determine the compatible topologies on $E$ for which $\left(e_{i}\right)_{i}$ is an equicontinuous Schauder basis.

This problem was studied by many Mathematcians, in particular by De Grande-De Kimpe [10]. It is also proved in ([22], 3.2. see also [32], 2.1 and $[12], 2.1)$ that in a $l K c s(E, \tau)$ there exists the finest locally $K$-convex topology $\nu$ of countable type compatible with $\tau$. The existence of this topology was also proved in ([21], proposition 2, p. 153). Thus, we are going to make, in the case of lKcs $(E, \tau)$ such that $E_{\sigma}=\left(E, \sigma\left(E, E^{\prime}\right)\right)$ and $E_{\sigma}^{\prime}=\left(E^{\prime}, \sigma\left(E^{\prime}, E\right)\right)$ are sequentially complete, this topology in relation with the original topology of $E$, by distinguishing the following three cases: $K$ is local, $K$ is spherically complete and $K$ is not spherically complete; which will give us a complete solution of this problem, we'll give a characterization of polar toplogies for which the weak basis problem is true in the case when $K$ is not spherically complete. We should remind that the problem of the weak basis was formulated by several ways and that's one of them ([21], p. 150)

(2) Is every weak Schauder basis for $E$ a Schauder basis for $E$ ?

We shall say that for a $l K c s E$ the weak basis theorem holds if every weak Schauder basis in $E$ is a Schauder basis .

In archimedean analysis, the weak basis theorem was first given for Banach spaces in 1932 by Banach ([3], p. 238) and extended to $(F)$-spaces 
by Bessaga and Pełczynski [5] (a $(F)$ - space is a complete, metrizable topological vector space. McArthur [24] proved an analogue for bases of subspaces in Frechet spaces. Arsove and Edwards [1] proved that the answer is positive if $E$ is a barrelled space. Singer shows by an example ([33], p. 153) that a weak basis need not be Schauder basis. Dubinsky and Retherford [15] observed that the answer is negative in general. Bennet and Cooper [4] proved it for strict $(L F)$ - spaces and Floret [17] for sequentially retractive $(L F)$ - spaces. M. De wilde [13] obtained a rather general result for bornological, sequentially complete and webbed spaces. W J. Stiles ([35], corollary 4.5, p. 413) showed that the theorem fails in non locally convex spaces $l^{p}(0 \prec p \prec 1)$. N. J. Kalton [20] gave a class of spaces for which this theorem is true. Joel H. Shapiro ([29], theorem 1, p. 1294) gave the following generalization of stiles'result

The weak basis theorem fails in every locally bounded non locally convex $(F)$-space which has a weak basis; he also gave a wide class of space which the weak basis theorem fails and proved the same for the space $H^{p}\left(H^{p}:=\right.$ the linear space of functions $f$ analytic in the open unit disc $|z| \prec 1$ such that $\left.\|f\|_{p}^{p}=\sup _{0 \leq r \prec 1} \int_{0}^{2 \pi}\left|f\left(r e^{i t}\right)\right|^{p} d t \prec \infty\right)$. Efimova [16] proved the weak basis theorem for regular inductive limits of a sequence of normed barrelled spaces. M. Valdivia has shown the result for metrizable barrelled spaces. J. Orihuela [27] gave a result which showed the linking between the weak basis theorem and the closed graph theorem. In [10] N. De Grande-De Kimpe solved completely the weak basis problem for locally convex space (lcs) $E$ having a $\sigma\left(E^{\prime}, E\right)$ - sequentially complete topological dual $E^{\prime}$.

In $n . a$ analysis, the weak basis problem has the following simple solution [21]: For a $l K c s E$ with a weak Schauder basis the weak basis theorem holds iff $E$ is an Orlicz-Pettis-space (a space, where every weakly convergent sequence is convergent). We know that, if $K$ is spherically complete then every $l K c s E$ is an $O P$-space; so the weak basis theorem holds in this case. If $K$ is not spherically complete J. Ka̧kol and T.Gilsdorf ([21], corollary 6, p. 155) proved that the weak basis theorem holds if $E$ is a polarly barrelled polar space (a locally $K$-convex space is called polarly barrelled if every closed, polar and absorbing absolutely $K$-convex subset of $E$ is a zeroneighbourhood), they provided a wide class of non-polar spaces $E$ with a weak Schauder basis which is a basic sequence in the original topology of $E$ ([21], example 7, p. 155 and 156). Finally J. Kạkol and T.Gilsdorf remark that they do not know if the following result is true: Let $E$ be a Banach 
space with a weak Schauder basis; then, $E$ is a polar space iff every weak Schauder basis in $E$ is a basic sequence ([21], remark 13, p. 160). This conjecture was set by P. Garcia and Schikhof ([28], p. 233) in the form of the following question: Does there exist a polar Banach space which is not $O P$-space, and does not contain $l^{\infty}$ ? . The answer of this question is negative; in the other words, the conjecture is true (see proposition 33).

In $\S$. 2 we'll give general results that are related to $l K c s$, to polar toplogies of $\mathcal{A}$-convergence and to space of sequences. In $\S$. 3 we study the perfect sequence spaces over $K$, we give a characterization of the natural topology noted $\mathrm{Na}$ with the sets absolutely $K$-convex, weakly bounded and compactoid if $K$ is spherically complete, with the sets absolutely $K$ convex and compact if $K$ is local and with the sets absolutely $K$-convex weakly bounded and $\Lambda$-closed ( $\Lambda$ is a perfect sequence space on $K$ ) when $K$ is not spherically complete. We are interested in $\S$. 4 in the study of transfer of topologies between a perfect sequence spaces and a $l K c s(E, \tau)$ that has a Schauder basis, using the two following algebraic isomorphisms

$\Phi: E \longrightarrow \Lambda \quad x \underset{l}{\longmapsto}\left(\lambda_{i}\right)_{i}$ and $\Psi: E^{\prime} \longrightarrow \Delta f \longmapsto\left(\mu_{i}\right)_{i}$ for every
$x=\sum_{i=1}^{\infty} \lambda_{i} e_{i}$ and $f=\sum_{i=1}^{\infty} \mu_{i} f_{i} ;$ where $\left(f_{i}\right)_{i}$ is the weak Schauder basis of $E^{\prime}$ associated to the Schauder basis $\left(e_{i}\right)_{i}$ of $E$ ([9], lemma 3, p. 402) and $\Lambda$ and $\Delta$ are two sequence spaces which we'll define like in [9]. This study will allow us to solve the problem (1) by distinguishing the three cases $K$ is local, $K$ is spherically complete and $K$ is not spherically complete. Some results that are related to problem (2) are given in the $\S .5$ by considering a polar $l K c s(E, \tau)$ which has a weak Schauder basis $\left(e_{i}\right)_{i}$ and as $E_{\sigma}$ and $E_{\sigma}^{\prime}$ are sequentially complete, we characterize the finest compatible topology on $E$ for which $\left(e_{i}\right)_{i}$ is a Schauder basis; this basis is necessary equicontinuous. Then, we give a necessary and sufficient condition which the topology $\tau$ must fulfill so as to admit $\left(e_{i}\right)_{i}$ as Schauder basis in the case when $K$ is non spherically complete. Then we deduce a new characterization of $O P$-spaces.

Finally, in $\S$. 6 we give applications to G-spaces and to $K$-barrelled spaces. We show that the result established by N. De Grande- De Kimpe in [10] in the classical case, for the barrelled spaces, is also true in the non archimedian case. For a $G$-space $(E, \tau)$; we show that $\tau$ is the only topology on $E$ compatible with the duality $\left\langle E, E^{\prime}\right\rangle$ for which $\left(e_{i}\right)_{i}$ is an equicontinuous Schauder basis. 


\section{Preliminaries}

1. Throughout $K$ is a non-archimedean (n.a) non trivially valued complete field with the valuation $|$.$| , and the valuation ring is B(0,1)$ $=\{\lambda \in K:|\lambda| \leq 1\}$.

2. Let $E$ be a $K$-vector space. A subset $A$ of $E$ is absolutely $K-$ convex if it is $B(0,1)-$ module. For a set $X \subset E$ its absolutely $K$-convex hull $\Gamma(X)$ is the smallest absolutely $K$-convex set containing $X$.

3. A topology on a vector space $E$ over $K$ is said to be locally $K-$ convex if there exists in $E$ a fundamental system of zero-neighbourhoods consisting of absolutely $K$-convex subsets of $E$.

In this paper the letter $E$ will always stand for Hausdorff locally $K-$ convex space over a field $K$.

4. A subset $A$ of $E$ is called compactoid if for every zero-neighbourhood $U$ in $E$, there exists a finite set $F \subset E$ such that $A \subset \Gamma(F)+U$.

5. A subset $A$ of $E$ is called c-compact if every convex filter on $A$ has a cluster point on $A$.

- An absolutely $K$-convex subset of a locally $K$-convex space $E$ is called $K$-closed if for every $x \in E$ the set $\{|\lambda| / \lambda \in K: \lambda x \in A\}$ is closed in $|K|$; the $K$-closed hull of $A$ is the smallest subset of $E$ which is $K$-closed and contains $A$, it is denoted by $K_{c}(A)$.

6. A sequence $\left(e_{i}\right)_{i}$ is a Schauder basis of $E$ if every $x \in E$ can be written uniquely as $x=\sum_{i=1}^{\infty} \lambda_{i} e_{i}$ where the coefficient functionals $f_{n}: x=$ $\sum_{i=1}^{\infty} \lambda_{i} e_{i} \longmapsto \lambda_{n}$ are continuous.

- The sequence $\left(f_{n}\right)_{n}$ is called the weak Schauder basis associated to basis $\left(e_{i}\right)_{i}$.

- For every $n \geq 1$, let $p_{n}$ the map $x=\sum_{i=1}^{\infty} \lambda_{i} e_{i} \longmapsto \lambda_{n} e_{n}$; the Schauder basis $\left(e_{i}\right)_{i}$ is called equicontinuous if the sequence $\left(p_{n}\right)_{n}$ is equicontinuous on $E$, this is equivalent to the equicontinuity of the sequence $\left(S_{n}\right)_{n}$ where $S_{n}: x=\sum_{i=1}^{\infty} \lambda_{i} e_{i} \longmapsto \sum_{i=1}^{n} \lambda_{i} e_{i}$, for every $n \geq 1$.

7. Let $\langle$,$\rangle be a duality between E$ and $F$ where $E$ and $F$ are two vectors spaces over $K$ (see [2] for general results);

- If $A$ is a subset of $E$, the polar of $A$ is a subset of $F$ defined by: $A^{\circ}=\{y \in F /|\langle x, y\rangle| \leq 1$ for all $x \in A\}$. 
We define also the polar of a subset $B$ of $F$ in the same way.

- The weak topology $\sigma(E, F)$ on $E$ is noted simply $\sigma$ and $\left\{A^{\circ} / A \in \mathcal{F}\right\}$ is a zero-neighbourhood base, where $\mathcal{F}$ is the set of finite subset of $F$.

- A subset $A$ of $F$ is said to be $E$ - closed if for every $y \in F \backslash A$, there exists $x \in E$ such that $|\langle x, y\rangle| \succ 1$ and $|\langle x, A\rangle| \leq 1$; the $E$-closed hull $E_{c}(A)$ of $A$ is the smallest $E$-closed subset of $F$ containing $A$.

Proposition 1. Let $A$ be an absolutely $K$-convex subset of $F$, then $A$ is $E$-closed, if and only if, $A$ is $K$-closed and $\sigma(F, E)-$ closed.

Proof. By [2], theorem 4.2, p. 233, proposition 2.5, p. 224 and corollary 4.3, p. 233.

8. Let $\mathcal{A}$ be a family of $\sigma(F, E)$-bounded subsets of $F$ such that

(a) $\mathcal{A}$ is directed by inclusion,

(b) $F=\bigcup_{A \in \mathcal{A}} A$,

(c) there exists $\lambda_{0} \in K,\left|\lambda_{0}\right|>1$, such that $\lambda_{0} A \in A$, for all $A \in \mathcal{A}$.

A topology $\tau$ on $E$ is called polar topology of $\mathcal{A}$ - convergence, if $\tau$ has a fundamental system of zero-neighbourhoods consisting of $\left\{A^{\circ} / A \in \mathcal{A}\right\}$

- A vector topology $\tau$ on $E$ is called polar topology if there exists a family $\mathcal{A}$ of $\sigma(F, E)$-bounded subsets of $F$ which has the properties $(a),(b)$ and (c), such that $\tau$ is a polar topology of $\mathcal{A}$-convergence.

- If $\tau$ is a polar topology of $\mathcal{A}$-convergence on $E$, it is determined by the family of n.a seminorms $\left(p_{A}\right)_{A \in \mathcal{A}}$, where $p_{A}(x)=\sup \{|\langle x, y\rangle| / y \in A\}([10]$, p. 277).

- If $\mathcal{A}$ is the family of all subsets of $F$ that are:

1. Absolutely $K$-convex, weakly bounded and weakly c-compacts, we have the c-compact topology $\tau_{c}(E, F)=\tau_{c}$,

2. Absolutely convex and $\sigma(F, E)$-compact, we have the Mackey topology $\tau_{m}(E, F)=\tau_{m}$,

3. $\sigma(F, E)$-bounded and $E$-closed, we have the $E$ - closed topology $\tau_{e}(E, F)=\tau_{e}$.

9. A locally $K$-convex topology $\tau$ on $E$ is called compatible with the duality $\langle E, F\rangle$ or $(E, F)$ - compatible if, $F$ is isomorphic to the topological dual of $E$ provided with the topology $\tau . \sigma(E, F)$ is the smallest of $(E, F)-$ compatible topology.

- A sequence $\left(e_{n}, f_{n}\right)_{n}$ of $E \times F$ is called biorthogonal if $\left\langle e_{n}, f_{n}\right\rangle=\delta_{n m}$, for all $n, m$ where $\delta_{n m}$ is the Kronecker delta.

10. The space of all sequences in $K$ is denoted by $\omega$, it is provided with the product topology $\tau_{\omega}$. A linear subspace of $\omega$ is called a sequence space. 
$\varphi, c_{0}$ and $l^{\infty}$ are respectively, the space of all sequences in $K$ with only finitely many non-zero terms, the space of the sequences in $K$ converging to zero and the space of the bounded sequences in $K$.

- for all $n \geq 1, e^{n}=\left(\delta_{n m}\right)_{m}$.

- Let $A \subset \omega$, the $\beta$-dual of $A$ is the subset $A^{\beta}$ of $\omega$ defined as $A^{\beta}=\left\{\lambda=\left(\lambda_{i}\right) \in \omega / \lim _{i} \lambda_{i} \alpha_{i}=0\right.$, for all $\left.\alpha=\left(\alpha_{i}\right)_{i} \in A\right\}$.

- $A$ is called $\beta$-perfect (or perfect) if $A=A^{\beta \beta}$.

- $A$ is solid if whenever $\left(a_{n}\right)_{n} \in A$ and $\left(\lambda_{n}\right)_{n} \in \omega$ such that $\left|\lambda_{n}\right| \leq 1$ for each $n$, then $\left(\lambda_{n} a_{n}\right)_{n} \in A$. The spaces $\omega, \varphi$ and $c_{0}$ are solids.

- The smallest solid subset of $\omega$ containing $A$ is called the solid hull of $A$, it is denoted by $\hat{A}$; and we have

$\hat{A}=\left\{\left(\lambda_{n} a_{n}\right)_{n} /\left(a_{n}\right)_{n} \in A\right.$ and $\left(\lambda_{n}\right)_{n} \in \omega:\left|\lambda_{n}\right| \leq 1$ for all $\left.n \geq 1\right\}$.

- Let $X$ be a sequence space in $K ; A \subset X$ is called solid in $X$ if $\hat{A} \cap X=A$. $\hat{A} \cap X$ is called the solid hull of $A$ in $X$.

- A topology on a vector space $X$ is called solid if there exists in $X$ a fundamental system of zero neighbourhoods consisting of solids subsets in $X$.

11. A G-space is a locally $K$-convex space $(E, \tau)$ such that $E^{\prime}$ is $\sigma\left(E^{\prime}, E\right)$ - sequentially complete and $\tau=\tau_{c}\left(\operatorname{resp} . \tau_{e}, \tau_{m}\right)$ if $K$ is spherically complete, ( resp. not spherically complete, local). In the last case, we find the notion of G-space given and studied by N. De Grande-De Kimpe in [11] in the classical case $(K=\mathbb{R}$ or $K=\mathscr{C})$.

\section{The natural topology in a perfect sequence spaces}

Let $\Lambda$ be a sequence space over $K$ containing $\varphi$, we consider the duality $\left\langle\Lambda, \Lambda^{\beta}\right\rangle$ defined by: $\left(\left(\lambda_{n}\right)_{n},\left(\mu_{n}\right)_{n}\right) \longmapsto\left\langle\left(\lambda_{n}\right)_{n},\left(\mu_{n}\right)_{n}\right\rangle=\sum_{n=1}^{\infty} \lambda_{n} \mu_{n}$ for every $\left(\lambda_{n}\right)_{n} \in \Lambda$ and $\left(\mu_{n}\right)_{n} \in \Lambda^{\beta}$.

For every $\mu=\left(\mu_{n}\right)_{n} \in \Lambda^{\beta}$, let $\hat{p}_{\mu}$ the n.a seminorm defined as $\hat{p}_{\mu}(\lambda)=$ $\sup \left|\lambda_{n} \mu_{n}\right|$, For every $\lambda=\left(\lambda_{n}\right)_{n} \in \Lambda$.

We call the locally $K$-convex topology on $\Lambda$ determined by the family of seminorms $\left(\hat{p}_{\mu}\right)_{\mu \in \Lambda^{\beta}}$ the natural topology; it will be denoted by $N a$.

Remark 1. The weak topology $\sigma$ on $\Lambda$ is weaker than the natural topology $N a$.

Proposition 2. If $\Lambda$ is perfect, then it is weakly sequentially complete. 
Proof. $\quad([25], 5.2, p .1550)$.

Proposition 3. If $\Lambda$ is perfect then every $\sigma$-bounded subset of $\Lambda$ is $\tau_{b}$-bounded, where $\tau_{b}$ is the strong topology $\tau_{b}\left(\Lambda, \Lambda^{\beta}\right)$ on $\Lambda$.

Proof. $\quad([8]$, proposition $8, p .476)$.

Corollary 1. If $\Lambda$ is perfect, all polars topologies on $\Lambda$ yield the same bounded sets.

Lemma 1. The solid hull of a finite subset of $\Lambda^{\beta}$ is $\sigma$-bounded.

Proof. Obvious.

Lemma 2. Let $A$ be a $\sigma$-bounded and solid subset of $\Lambda^{\beta}$, then the polar of $A$ in the duality $\left\langle\Lambda, \Lambda^{\beta}\right\rangle$ is given by $A^{\circ}=\left\{\lambda \in \Lambda / \hat{p}_{\mu}(\lambda) \leq 1\right.$, for all $\left.\mu \in A\right\}$.

Proof. ([8], proposition 1, p. 472).

Proposition 4. The natural topology on $\Lambda$ is a polar topology.

Proof. Obvious.

Remark 2. The natural topology is a solid topology; in fact it is the coarsest of the polar and solid topologies on $\Lambda$.

Proposition 5. If $\Lambda$ is perfect, the natural topology $N a$ is compatible with the duality $\left\langle\Lambda, \Lambda^{\beta}\right\rangle$.

Proof. The $N a$ topology is polar and for every $\mu \in \Lambda^{\beta},(\{\hat{\mu}\})^{\circ}=$ $\left[K_{c}\left(\overline{\{\hat{\mu}\}}^{\sigma\left(\Lambda^{\beta}, \Lambda\right)}\right)\right]^{\circ}[2]$, corollary 4.3, p. 233* Then, if we take $\mathcal{A}=\left\{K_{c}\left(\overline{\hat{A}}^{\sigma\left(\Lambda^{\beta}, \Lambda\right)}\right) / A \subset \Lambda^{\beta}\right.$ and A is finite $\}$ so $N a$ is a polar topology of $\mathcal{A}$-convergence, where $\mathcal{A}$ is formed by a $\sigma\left(\Lambda^{\beta}, \Lambda\right)$ - bounded and $\Lambda$ closed subsets of $\Lambda^{\beta}$ (proposition 1 ). Then by [2], theorem 4.3, p. 233 the natural topology $N a$ is compatible.

Remark 3. For every $\mu \in \Lambda^{\beta}, \overline{\Gamma(\{\hat{\mu}\})}$ is weakly-c-compact if $K$ is spherically complete and weakly compact if $K$ is local. 
Proposition 6. If $\Lambda$ is perfect, then it is complete under any polar solid topology.

Proof. Let $\tau$ be a solid polar topology of $\mathcal{A}$-convergence on $\Lambda$; we consider $\left(\lambda^{i}\right)_{i \in I}$ as a Cauchy-net in $(\Lambda, \tau)$.

Let $A \in \mathcal{A}$, there exists $i_{0} \in I$ such that $\lambda^{i}-\lambda^{j} \in A^{\circ}$, for all $i, j \geq i_{0}$; so we have (1) $\sup _{\alpha=\left(\alpha_{n}\right)_{n} \in A} \sup _{n}\left|\alpha_{n}\left(\lambda_{n}^{i}-\lambda_{n}^{j}\right)\right| \leq 1$, for all $i, j \geq i_{0}$ (lemma 2).

Then for every $n,\left(\lambda_{n}^{i}\right)_{i \in I}$ is a Cauchy-net in $K$, so there exists $\lambda_{n} \in K$ such that $\lambda_{n}=\lim _{i} \lambda_{n}^{i}$. Therefore, from (1) we obtain:

(2) $\sup _{\alpha=\left(\alpha_{n}\right)_{n} \in A} \sup _{n}\left|\alpha_{n}\left(\lambda_{n}^{i}-\lambda_{n}\right)\right| \leq 1$, for all $i \geq i_{0}$.

Let $\alpha=\left(\alpha_{n}\right)_{n} \in \Lambda^{\beta}$, there exists $A \in \mathcal{A}$ such that $\alpha \in A$ and we have for all $n \geq 1,\left|\alpha_{n} \lambda_{n}\right| \leq \max \left(\left|\lambda_{n}^{i_{0}} \alpha_{n}\right|,\left|\left(\lambda_{n}^{i_{0}}-\lambda_{n}\right) \alpha_{n}\right|\right)$.

Hence $\lambda=\left(\lambda_{n}\right)_{n} \in \Lambda^{\beta \beta}=\Lambda$ ( $\Lambda$ is perfect) and by (2) we have $\lambda=$ $\lim _{i} \lambda^{i}$ in $(\Lambda, \tau)$.

Proposition 7. Let $A$ be a subset of $\Lambda$; if $A$ is $N a$-bounded then $\hat{A}$ is Na-bounded.

Proof. Obvious.

Proposition 8. Suppose that $\Lambda$ is perfect and let $\tau$ be a polar topology on $\Lambda$ and $A$ be a subset of $\Lambda$. If $A$ is $\tau$-bounded, then $\hat{A}$ is $\tau$-bounded.

Proof. $\quad A$ is $\tau$-bounded $\Rightarrow A$ is $N a$-bounded (corollary 1 and proposition 4$) \Rightarrow \hat{A}$ is $N a$-bounded (proposition 7) $\Rightarrow \hat{A}$ is $\tau$-bounded (corollary 1 and proposition 4$)$.

Corollary 2. $\tau_{b}$ is a solid topology.

Proof. $\quad \Lambda^{\beta}$ is perfect, then for every $A \subset \Lambda^{\beta}, A$ is $\sigma$-bounded $\Longleftrightarrow \hat{A}$ is $\sigma$-bounded.

Lemma 3. Let $E$ and $F$ be a locally $K$-convex spaces and $A$ a compactoid subset of $E$. If $\left(f_{n}\right)_{n \geq 1}$ is an equicontinuous sequence of linear mappings from $E$ to $F$ pointwise converging to a mapping $f$, then $\left(f_{n}\right)_{n \geq 1}$ converges to $f$ uniformly on $A$.

Proof. ([8], proposition 13, p. 477). 
Remark 4. The lemma before is true if we replace compactoid by precompact (if $K$ is local) or bounded and c-compact (if $K$ is spherically complete).

Proposition 9. Let $A$ be a compactoid subset of $(\Lambda, N a)$. Then for every $\alpha=\left(\alpha_{n}\right)_{n} \in \Lambda^{\beta}, \lim _{k}\left|\alpha_{k}\right| \sup _{\lambda \in A}\left|\lambda_{k}\right|=0$.

Proof. Let $\alpha=\left(\alpha_{n}\right)_{n} \in \Lambda^{\beta}$; for every $n \in \mathbb{N}$, we consider $\alpha^{n}=$ $\alpha_{n} e^{n}$ with $e^{n}=\left(\delta_{n m}\right)_{m}$. Then for every $\mu=\left(\mu_{n}\right)_{n} \in \Lambda, \hat{p}_{\mu}\left(\alpha^{n}\right)=$ $\left|\mu_{n} \alpha_{n}\right| \stackrel{n \rightarrow \infty}{\longrightarrow} 0$, so $\lim _{n \rightarrow \infty} \alpha^{n}=0$ in $\left(\Lambda^{\beta}, N a\right)$. On the other hand $\left(\alpha^{n}\right)_{n}$ is $N a$-equicontinuous ([8], proposition 3, p. 474); then according to lemma before $\left(\alpha^{n}\right)_{n}$ converges to 0 uniformly on $A$.

Remark 5. Suppose that $K$ is spherically complete and let $\tau$ be a locally $K$-convex topology compatible with the duality $\left\langle\Lambda, \Lambda^{\beta}\right\rangle$ on $\Lambda$ and $A$ an absolutely $K$-convex bounded and c-compact subset of $\Lambda$ in $(\Lambda, \tau)$. Then for every $\alpha \in \Lambda^{\beta}, \lim _{k}\left|\alpha_{k}\right| \sup _{\lambda \in A}\left|\lambda_{k}\right|=0$.

Proof. Remark 4, proposition 5, [36] theorem 4.21 and [7] proposition 3.

Proposition 10. The sequence $\left(e^{n}\right)_{n}$ is a Schauder basis of $(\Lambda, N a)$.

Proof. Let $\lambda=\left(\lambda_{n}\right)_{n} \in \Lambda$, then for every $\mu=\left(\mu_{n}\right)_{n} \in \Lambda^{\beta}, \hat{p}_{\mu}\left(\lambda_{i} e^{i}\right)=$ $\left|\lambda_{i} \mu_{i}\right| \stackrel{i \rightarrow \infty}{\longrightarrow} 0$. Therefore $\sum_{i} \lambda_{i} e^{i}$ converges in $(\Lambda, N a)$ and so every element $\lambda=\left(\lambda_{n}\right)_{n} \in \Lambda$ can be written uniquely as $\lambda=\sum_{n=1}^{\infty} \lambda_{n} e^{n}$. On the other hand, for every $n \in \mathbb{I}, e^{n} \in \Lambda^{\beta}$ and we have $\hat{p}_{e^{n}}(\lambda)=\left|\lambda_{n}\right|$ for all $\lambda=\left(\lambda_{n}\right)_{n} \in \Lambda$, hence the maps $\lambda=\sum_{i=1}^{\infty} \lambda_{i} e^{i} \longmapsto \lambda_{n}$ is $N a$-continuous.

Theorem 1. Suppose that $K$ is spherically complete and $\Lambda$ is perfect. A subset $A$ of $\Lambda$ is compactoid in $\Lambda_{N a}$ if, and only if, it is a subset of the solid hull of a singleton of $\Lambda$.

Proof. $\Longrightarrow$ Let $A$ be a compactoid subset of $\Lambda_{N a}$.

Let $\varrho>1$ and $\lambda=\left(\lambda_{n}\right)_{n} \in \omega$ such that $\left|\lambda_{n}\right|=\varrho^{n}$ for all $n \geq 1$. 
$A$ is compactoid in $\Lambda_{N a}$, so it is $N a$-bounded, and therefore sup $\left|\alpha_{i}\right|<$ $\left(\alpha_{i}\right)_{i} \in A$

$+\infty$, for all $i \geq 1$, there exists $n_{i} \geq 1$ such that $\varrho^{n_{i}-1} \leq \sup _{\left(\alpha_{i}\right) \in A}\left|\alpha_{n}\right| \leq \varrho^{n_{i}}$.

Let $\mu=\left(\mu_{i}\right)_{i}$ the element of $\omega$ given by $\mu_{i}=\lambda_{n_{i}}$ for all $i \geq 1$, then for every $\alpha=\left(\alpha_{i}\right)_{i} \in \Lambda^{\beta}$ we have: for all $i \geq 1$,

$\left|\alpha_{i}\right| \sup _{\left(\gamma_{i}\right) \in A}\left|\gamma_{i}\right|$

$$
\left|\mu_{i} \alpha_{i}\right| \leq\left|\alpha_{i}\right|\left(\varrho^{n_{i}}-\sup _{\left(\gamma_{i}\right) \in A}\left|\gamma_{i}\right|\right)+\left|\alpha_{i}\right| \sup _{\left(\gamma_{i}\right) \in A}\left|\gamma_{i}\right| \leq \varrho
$$

Now, $\lim _{i}\left|\alpha_{i}\right| \sup _{\left(\gamma_{i}\right)_{i} \in A}\left|\gamma_{i}\right|=0$ (proposition 9), hence $\lim _{i} \mu_{i} \alpha_{i}=0$. Then $\mu \in \Lambda^{\beta \beta}=\Lambda^{*}$ On the other hand, if $\alpha=\left(\alpha_{i}\right)_{i} \in A$, we have $\left|\alpha_{i}\right| \leq\left|\mu_{i}\right|$, for every $i \geq 1$, hence $\alpha \in\{\hat{\mu}\}$.

$\Longleftarrow]$ It suffices to prove that $\{\hat{\lambda}\}$ is compactoid in $\Lambda_{N a}$, for every $\lambda \in \Lambda$.

Let $\lambda=\left(\lambda_{n}\right)_{n}$ an element of $\Lambda$, then for every $\alpha=\left(\alpha_{n}\right)_{n}$ in $\Lambda^{\beta}$, there exists $n_{0} \in \mathbb{N}$ such that $\left|\lambda_{n} \alpha_{n}\right| \leq 1$ for all $n>n_{0}$. We put $\lambda^{i}=\lambda_{i} e^{i}$, for all $i, 1 \leq i \leq n_{0}$.

If $\mu=\left(\mu_{i} \lambda_{i}\right)_{i}$ is an element of $\{\hat{\lambda}\}, \mu=\sum_{i=1}^{\infty} \mu_{i} \lambda_{i} e^{i}=\sum_{i=1}^{n_{0}} \mu_{i} \lambda^{i}+\sum_{i>n_{0}} \mu_{i} \lambda_{i} e^{i}$.

Now, $\hat{p}_{\alpha}\left(\sum_{i>n_{0}} \mu_{i} \lambda_{i} e^{i}\right)=\sup _{i>n_{0}}\left|\mu_{i} \lambda_{i} \alpha_{i}\right| \leq 1$

So $\mu \in \Gamma\left(\lambda^{1}, \lambda^{2}, \ldots, \lambda^{n_{0}}\right)+B_{\hat{p}_{\alpha}}(0,1)$, where $B_{\hat{p}_{\alpha}}(0,1)=\left\{\lambda / \hat{p}_{\alpha}(\lambda) \leq 1\right\}$.

Then $\{\hat{\lambda}\} \subset \Gamma\left(\lambda^{1}, \lambda^{2}, \ldots, \lambda^{n_{0}}\right)+B_{\hat{p}_{\alpha}}(0,1)$.

Remarks 1. 1. N. De Grande-De Kimpe gave an analogue proposition of theorem in which she characterize the weakly c-compact subsets ([8], proposition 15, p. 478 ). This proposition is in fact true for all compatible topologies with the duality $\left\langle\Lambda, \Lambda^{\beta}\right\rangle$ in particular for the natural topology $N a$.

2. $K_{c}\left(\overline{\{\hat{\mu}\}}^{\sigma\left(\Lambda, \Lambda^{\beta}\right)}\right)$ is compactoid for every field $K$.

Consequently the solid hull of a bounded subset which is absolutely $K$ - convex and c-compact of $\Lambda$ is also bounded and c-compact for every compatible topology.

Corollary 3. i. For every $\lambda \in \Lambda^{\beta},\{\hat{\lambda}\}$ is compactoid in $\left(\Lambda^{\beta}, N a\right)$ for every field $K$. 
ii. Suppose that $\Lambda$ is perfect and $K$ is spherically complete and let $A$ be a subset of $\Lambda$. Then $A$ is compactoid in $\Lambda_{N a}$ if, and only if, there exists a sequence $\left(\alpha^{n}\right)_{n}$ converging to zero in $\Lambda_{N a}$ such that $A \subset \overline{\Gamma\left(\alpha^{1}, \alpha^{2}, \ldots, \alpha^{n}, \ldots\right)}$.

Proof. i. $\Lambda^{\beta}$ is perfect; it suffices to use the theorem 1.

ii. Let $\lambda=\left(\lambda_{n}\right)_{n} \in \Lambda$ such that $A \subset\{\hat{\lambda}\}$ (theorem 1$)$. We put $\alpha^{n}=$ $\lambda_{n} e^{n}$ for all $n \geq 1$, then for every $\mu=\left(\mu_{n}\right)_{n} \in \Lambda^{\beta}$ we have $\hat{p}_{\mu}\left(\alpha^{n}\right)=$ $\left|\mu_{n} \lambda_{n}\right| \stackrel{n \rightarrow \infty}{\longrightarrow} 0$, so the sequence $\left(\alpha^{n}\right)_{n}$ is converging to zero in $\Lambda_{N a}$. On the other hand for every $a=\left(a_{n}\right)_{n} \in A, a \in\{\hat{\lambda}\}$, therefore there exists $\left(\mu_{n}\right)_{n} \in$ $\omega$ such that $\left|\mu_{n}\right| \leq 1$ for all $n$ and $a=\left(\mu_{n} \lambda_{n}\right)_{n} ;$ so $a=\sum_{n=1}^{\infty} \mu_{n} \alpha^{n}$ (proposition 10). Then $a \in \overline{\Gamma\left(\alpha^{1}, \alpha^{2}, \ldots, \alpha^{n}, \ldots\right)}$.

Conversely, suppose that $A \subset \overline{\Gamma\left(\alpha^{1}, \alpha^{2}, \ldots, \alpha^{n}, \ldots\right)}$ where $\left(\alpha^{n}\right)_{n}$ converges to zero in $\Lambda_{N a}$. Let $U$ be a zero-neighbourhood in $\Lambda_{N a}$, then there is $n_{0} \in \mathbb{N}$ such that for all $n>n_{0}, \alpha^{n} \in U$. So $\Gamma\left(\alpha^{1}, \alpha^{2}, \ldots, \alpha^{n}, \ldots\right) \subset$ $\Gamma\left(\alpha^{1}, \alpha^{2}, \ldots, \alpha^{n_{0}}\right)+U$, (we can choose $U$ absolutely $K$-convex and open). Then $\overline{\Gamma\left(\alpha^{1}, \alpha^{2}, \ldots, \alpha^{n}, \ldots\right)} \subset \Gamma\left(\alpha^{1}, \alpha^{2}, \ldots, \alpha^{n_{0}}\right)+U$, because $\Gamma\left(\alpha^{1}, \alpha^{2}, \ldots, \alpha^{n_{0}}\right)+U$ is $N a-$ closed.

\section{Characterization of the natural topology a- $K$ is spherically complete}

Theorem 2. If $\Lambda$ is perfect, then the natural topology on $\Lambda$ is the polar topology of $\mathcal{A}$-convergence, where $\mathcal{A}$ is the family of all compactoid subsets of $\left(\Lambda^{\beta}, N a\right)$.

Proof. Let $\mathcal{A}$ be the family of all compactoid subsets of $\left(\Lambda^{\beta}, N a\right)$ then for every $A \in \mathcal{A}, A$ is $\sigma-$ bounded and $\mathcal{A}$ satisfies the conditions $(a),(b)$ and $(c)$ of 8 . Let $\tau$ be the polar topology of $\mathcal{A}$ - convergence on $\Lambda$; then $\tau=N a$, (theorem 1) .

Theorem 3. If $\Lambda$ is perfect, then the natural topology on $\Lambda$ is the polar topology of $\mathcal{A}$-convergence where $\mathcal{A}$ is the family of all absolutely $K-$ convex, bounded and c-compact subsets of $\left(\Lambda^{\beta}, N a\right)$.

Proof. The same as theorem 2 and apply remarks 1.

Remark 6. If $K$ is spherically complete and $\Lambda$ is perfect then $N a=\tau_{c}$; where $\tau_{c}$ is the c-compact topology on $\Lambda$. And for every topology $\tau$ on $\Lambda$, $\tau$ is compatible with the duality $\left\langle\Lambda, \Lambda^{\beta}\right\rangle$ if, and only if, $\sigma \leq \tau \leq N a$. 


\section{b- $K$ is local}

If $K$ is local, then [31], proposition 1 and [7], proposition 2, p. 177 induce that all results before still hold when the word absolutely $K$-convex, bounded and c-compact (or compactoid) is replaced by absolutely $K$-convex and compact; and the characterization of the natural topology became:

Theorem 4. If $\Lambda$ is perfect, then the natural topology on $\Lambda$ is the polar topology of $\mathcal{A}$-convergence where $\mathcal{A}$ is the family of all absolutely $K-$ convex and compact subsets of $\left(\Lambda^{\beta}, N a\right)$.

\section{c- $K$ is not spherically complete}

Theorem 5. If $\Lambda$ is perfect, then the natural topology on $\Lambda$ is the polar topology of $\mathcal{A}$-convergence where

$\mathcal{A}=\left\{K_{c}\left(\overline{\hat{A}}^{\sigma\left(\Lambda^{\beta}, \Lambda\right)}\right) / A \subset \Lambda^{\beta}\right.$ and $A$ is finite $\}$.

Proof. By proposition 5.

\section{Locally $K$-convex spaces with a Schauder basis and per- fect sequence spaces}

Let $(E, \tau)$ be a locally $K$-convex space where $\tau$ is a polar topology of $\mathcal{A}$-convergence, $\left(e_{i}\right)_{i}$ be a Schauder basis of $(E, \tau)$ and $\left(f_{i}\right)_{i}$ the associated weak Schauder basis. If $S_{n}(x)=\sum_{i=1}^{n} \lambda_{i} e_{i}$ and $T_{n}(f)=\sum_{i=1}^{n} \mu_{i} f_{i}$ for all $x \in E$, all $f \in E^{\prime}$ (the topological dual of $E$ ) and all $n \geq 1,\left\langle S_{n}(x), f\right\rangle=$ $\left\langle x, T_{n}(f)\right\rangle$, for all $n \geq 1, x \in E$ and $f \in E^{\prime}$. For every $A \subset E^{\prime}$, we put $\widetilde{A}=\left\{T_{n}(a) / n \in \mathbb{N} ; a \in A\right\}$ with $T_{0}=i d_{E^{\prime}}$ and for every $A \subset E$ we put $S(A)=\left\{x \in A / S_{n}(x) \in A\right.$, for all $\left.n \geq 1\right\}$. We define also $\widetilde{A}$ for $A \subset E$ and $S(A)$ for $A \subset E^{\prime}$. N. De Grande-De Kimpe has defined the topology $\widetilde{\tau}$ of $\widetilde{\mathcal{A}}$-convergence where $\widetilde{\mathcal{A}}=\{\widetilde{A} / A \in \mathcal{A}\}$, and she gave a characterization of this topology ([10], proposition 1.2, p. 278). We enhance this result in theorem 6, p. 19.

Remarks 2. 1. $\widetilde{p}_{A}(x)=\sup _{n} p_{A}\left(\sum_{i=1}^{n}\left\langle x, f_{i}\right\rangle e_{i}\right)$ for all $A \in \mathcal{A}$ for all $x \in$ $E$; in the case where $F=E^{\prime}, p_{\widetilde{A}}(x)=\widetilde{p}_{A}(x)=\sup _{n} p_{A}\left(S_{n}(x)\right)=\sup _{n}$ $p_{A}\left(f_{n}(x) \cdot x_{n}\right)$ ([10], proposition 1.1, p. 278). 
2. The $\widetilde{\sigma}-$ topology associated to the weak topology $\sigma=\sigma\left(E, E^{\prime}\right)$ on $E$ is defined by the family of seminorms n.a $\left(p_{f}\right)_{f \in E^{\prime}}$, where $p_{f}(x)=$ $\sup _{n}\left|\left\langle S_{n}(x), f\right\rangle\right|$, for every $x \in E$ and $f \in E^{\prime}$; and we have $\widetilde{\sigma} \leq \widetilde{\tau}$.

Example 1. Let $\Lambda$ be a perfect sequence space over $K$. The topology $\widetilde{\sigma}=$ $\tilde{\sigma}\left(\Lambda, \Lambda^{\beta}\right)$ associated to $\sigma=\sigma\left(\Lambda, \Lambda^{\beta}\right)$ is defined by the family of seminorms n.a $\left(p_{\mu}\right)_{\mu \in \Lambda^{\beta}}$, where $p_{\mu}(\lambda)=\sup _{n}\left|\sum_{i=1}^{n} \lambda_{i} \mu_{i}\right|=\sup _{n}\left|\lambda_{n} \mu_{n}\right|$, for every $\lambda=$ $\left(\lambda_{i}\right)_{i} \in \Lambda$ and $\mu=\left(\mu_{i}\right)_{i} \in \Lambda^{\beta}$. Then the topology $\widetilde{\sigma}$ is exactly the natural topology studied in $\S$. 3 .

We Consider the two linear mappings $\Phi: E \longrightarrow \Lambda, x=\sum_{i=1}^{\infty} \lambda_{i} e_{i} \longmapsto$ $\left(\lambda_{i}\right)_{i}$ and $\Psi: E^{\prime} \longrightarrow \Delta, \quad f=\sum_{i=1}^{\infty} \mu_{i} f_{i} \longmapsto\left(\mu_{i}\right)_{i}$; where $\Lambda$ and $\Delta$ are the sequence spaces defined as $\Lambda=\left\{\left(\lambda_{i}\right)_{i} \in \omega / \sum_{i=1}^{\infty} \lambda_{i} e_{i}\right.$ converges in $\left.(E, \tau)\right\}$ and $\Delta=\left\{\left(\mu_{i}\right)_{i} \in \omega / \sum_{i=1}^{\infty} \mu_{i} f_{i}\right.$ converges in $\left.E_{\sigma}^{\prime}\right\} . \Phi$ and $\Psi$ are algebraic isomorphisms.

Proposition 11. $\Lambda \subset \Delta^{\beta}$ and $\Delta \subset \Lambda^{\beta}$.

Proposition 12. i. $\Phi$ is $\left(\sigma\left(E, E^{\prime}\right), \sigma(\Lambda, \Delta)\right)$ - continuous;

ii. $\Psi$ is $\left(\sigma\left(E^{\prime}, E\right), \sigma(\Delta, \Lambda)\right)$ - continuous.

Proof. $\quad$ i. Let $\mu \in \Delta$, we consider $V=\left\{\lambda=\left(\lambda_{i}\right)_{i} \in \Lambda /|\langle\lambda, \mu\rangle| \leq 1\right\}$. We put $f=\psi^{-1}(\mu)$, then $f \in E^{\prime}$. We Consider $U=\{x \in E /|\langle x, f\rangle| \leq 1\}$; $U$ is a zero neighbourhood in $\left(E, \sigma\left(E, E^{\prime}\right)\right)$, and we have $\Phi(U)=\{\lambda=\Phi(x) \in E /|\langle x, f\rangle| \leq 1\}=V$.

If $x=\sum_{i=1}^{\infty} \lambda_{i} e_{i}$ and $\mu=\left(\mu_{i}\right)_{i}$, then $\Phi(x)=\left(\lambda_{i}\right)_{i}$ and $f=\sum_{i=1}^{\infty} \mu_{i} f_{i}$.

Therefore $\langle x, f\rangle=\sum_{i=1}^{\infty} \lambda_{i} \mu_{i}=\langle\Phi(x), \mu\rangle$.

ii. Same proof as for i. 
Proposition 13. If $\Phi^{*}$ and $\Psi^{*}$ are the algebraic adjoints of $\Phi$ and $\Psi$ respectively, then $\Phi^{*}=\Psi^{-1}$ and $\Psi^{*}=\Phi^{-1}$.

Proof. $\Phi^{*}$ take his values in $E^{\prime}$ ( [30], p. 128 ). For every $x \in E$ and $\mu \in \Delta$ we have $\left\langle x, \Phi^{*}(\mu)\right\rangle=\langle\Phi(x), \mu\rangle=\sum_{i=1}^{\infty} \lambda_{i} \mu_{i}$, where $x=\sum_{i=1}^{\infty} \lambda_{i} e_{i}$ and $\mu=\left(\mu_{i}\right)_{i}$. So $\left\langle x, \Phi^{*}(\mu)\right\rangle=\left\langle x, \Psi^{-1}(\mu)\right\rangle$. Then $\Phi^{*}=\Psi^{-1}$.

The same for $\Psi^{*}=\Phi^{-1}$.

Proposition 14. a. For every $A \subset E,(\Phi(A))^{\circ}=\Psi\left(A^{\circ}\right)$;

b. For every $B \subset E^{\prime},(\Psi(B))^{\circ}=\Phi\left(B^{\circ}\right)$.

Proof. $\quad$ a. Let $A \subset E$, then $(\Phi(A))^{\circ}=\left(\Phi^{*}\right)^{-1}\left(A^{\circ}\right)[[2]$, proposition 2.8, p. 225].

Now $\Phi^{*}=\Psi^{-1}($ proposition 13$)$, so $(\Phi(A))^{\circ}=\left(\Psi^{-1}\right)^{-1}\left(A^{\circ}\right)=$ $\Psi\left(A^{\circ}\right)$.

b. The same proof.

The topology $\tau_{\Phi}$ defined on $\Lambda$ by $\Phi$ has a zero-neighbourhood base consisting of the family $\left(\Phi\left(A^{\circ}\right)\right)_{A \in \mathcal{A}}$ ([6], II. 29), $\tau_{\Phi}$ is a polar topology of $\Psi(\mathcal{A})$-convergence, where $\Psi(\mathcal{A})=\{\Psi(A) / A \in \mathcal{A}\}$ (proposition 14).

Examples 1. 1. If we consider the space $E_{\sigma}$, then the topology $\sigma_{\Phi}$ has a zero-neighbourhood base the set $\left\{(\psi(A))^{\circ} / A \subset E^{\prime}\right.$ and $A$ is finite $\}$.

For $A=\left\{\left(f^{i}\right)_{1 \leq i \leq n} / f^{i} \in E^{\prime}\right\}$, put $f^{i}=\sum_{j=1}^{\infty} \mu_{j}^{i} f_{j}$, for every $i, 1 \leq i \leq$ $n ;(\Psi(A))^{\circ}=\left(\left\{\left(\mu_{j}^{i}\right)_{j \geq 1}, 1 \leq i \leq n\right\}\right)^{\circ}$. Hence $\sigma_{\Phi}$ is exactly the weak topology.

2. Let $\tau$ be a polar topology of $\mathcal{A}$-convergence on $E$ and $\widetilde{\tau}$ the associated polar topology, then $\widetilde{\tau}_{\Phi}=(\widetilde{\tau})_{\Phi}$ has $\left[(\Psi(\widetilde{A}))^{\circ}\right]_{A \in \mathcal{A}}$ as a zero-neighbourhood base. For every $A \in \mathcal{A}, \Psi(\widetilde{A})=\widetilde{\Psi(A)}$, then $\widetilde{\tau}_{\Phi}$ is defined by the family of n.a seminorms $\left(\widetilde{p}_{\Psi(A)}\right)_{A \in \mathcal{A}}$, where :

$\widetilde{p}_{\Psi(A)}\left(\left(\lambda_{i}\right)_{i \geq 1}\right)=\sup _{n} p_{\psi(A)}\left(\lambda_{1}, \ldots, \lambda_{n}, 0, \ldots\right)=\sup _{n} \sup _{\mu=\left(\mu_{i}\right) \in A}\left|\sum_{i=1}^{n} \lambda_{i} \mu_{i}\right|=$ $\sup _{\mu=\left(\mu_{i}\right) \in A} \widetilde{p}_{\mu}\left(\left(\lambda_{i}\right)_{i \geq 1}\right)$. 
3. The direct image topology of $\widetilde{\sigma}$ with $\Phi$ on $\Lambda$, noted $\widetilde{\sigma}_{\Phi}$, is defined with the family of semi-norms n.a $\left(\widetilde{p}_{\Psi(f)}\right)_{f \in E^{\prime}}$, where $\widetilde{p}_{\Psi(f)}\left(\left(\lambda_{i}\right)_{i}\right)=$ $\widetilde{p}_{\mu}\left(\left(\lambda_{i}\right)_{i}\right),\left(\lambda_{i}\right)_{i} \in \Lambda$ and $\mu=\Psi(f)$. Then $\widetilde{\sigma}_{\Phi}$ is exactly the natural topology on $\Lambda$.

\section{Some properties of the topology $\widetilde{\sigma}$}

Lemma 4. i. If $E_{\sigma}$ is sequentially complete then $\Lambda=\Delta^{\beta}$;

ii. If $E_{\sigma}^{\prime}$ is sequentially complete then $\Delta=\Lambda^{\beta}$;

iii. If $E_{\sigma}$ and $E_{\sigma}^{\prime}$ are sequentially complete then $\Lambda$ is perfect.

Proof. $\quad$ i. $\Lambda \subset \Delta^{\beta}$ (proposition 11).

Let $\lambda=\left(\lambda_{i}\right)_{i}$ an element of $\Delta^{\beta}$, so $\lambda=\lim _{n \rightarrow \infty} \sum_{i=1}^{n} \lambda_{i} e^{i}$ in $\left(\Delta^{\beta} \sigma\left(\Delta^{\beta} \Delta^{\beta \beta}\right)\right)=$ $\Delta_{\sigma}^{\beta}$ where $e^{i}=\left(\delta_{i j}\right)_{j}$ for all $i \geq 1,\left(\Delta^{\beta}\right.$ is perfect $)$. Then $\left(e^{i}\right)_{i}$ is a Schauder basis of $\Delta_{\sigma}^{\beta}$ (propositions 5,10 and remark 1), so $\lambda=\lim _{n \rightarrow \infty} \sum_{i=1}^{n} \lambda_{i} e^{i}$ in $\left(\Delta^{\beta}, \sigma\left(\Delta^{\beta}, \Delta\right)\right) \cdot\left(\sum_{i=1}^{n} \lambda_{i} e^{i}\right)_{n}$ is a Cauchy-sequence in $(\Lambda, \sigma(\Lambda, \Delta))$ which is sequentially-complete (examples 1. 1), then $\lambda=\lim _{n \rightarrow \infty} \sum_{i=1}^{n} \lambda_{i} e^{i}$ in $(\Lambda, \sigma(\Lambda, \Delta)$ ) and so $\lambda \in \Lambda$.

ii. $\Delta \subset \Lambda^{\beta}$ (proposition 11). Let $\lambda=\left(\lambda_{i}\right)_{i} \in \Lambda^{\beta}$, for every $x \in E$, there exists $\alpha=\left(\alpha_{i}\right)_{i} \in \Lambda$ such that $x=\sum_{i=1}^{\infty} \alpha_{i} e_{i}$. for all $n \geq 1, \lambda_{n} f_{n}(x)=$ $\lambda_{n} \alpha_{n} \stackrel{n \rightarrow \infty}{\longrightarrow} 0$; then $\sum_{j \geq 1} \lambda_{j} f_{j}$ is convergent in $E_{\sigma}^{\prime}$; and so $\lambda=\left(\lambda_{i}\right)_{i} \in \Delta$.

Proposition 15. i. If $E_{\sigma}$ is sequentially complete then $E_{\widetilde{\sigma}}$ is complete;

ii. If $E_{\sigma}^{\prime}$ is sequentially complete then $E_{\widetilde{\sigma}}^{\prime}$ is complete; where $E_{\widetilde{\sigma}}=\left(E, \widetilde{\sigma}\left(E, E^{\prime}\right)\right)$ and $E_{\widetilde{\sigma}}^{\prime}=\left(E^{\prime}, \widetilde{\sigma}\left(E^{\prime}, E\right)\right)$.

Proof. i. Suppose that $E_{\sigma}$ is sequentially complete, then by lemma 4 $\Lambda=\Delta^{\beta}$ and $\left(\Lambda, \widetilde{\sigma}\left(\Lambda, \Delta^{\beta \beta}\right)\right)$ is complete ( proposition 6 and remark 2), then $(\Lambda, \widetilde{\sigma}(\Lambda, \Delta))$ is complete and $E_{\widetilde{\sigma}}$ is also complete (examples 1. 3).

ii. Same proof as for i.

Proposition 16. If $E_{\sigma}$ is sequentially complete and has an equicontinuous Schauder basis, then $E$ is isomorphic to a closed subspace of some power of $K$. 
Proof. Suppose that $E_{\sigma}$ is sequentially complete and admits an equicontinuous Schauder basis, then the topologies $\sigma\left(E, E^{\prime}\right)$ and $\widetilde{\sigma}\left(E, E^{\prime}\right)$ coincides on $E$, then by proposition $15\left(E, \sigma\left(E, E^{\prime}\right)\right)$ is complete; and the proposition follows ([7], proposition 7, p. 179).

Proposition 17. If $E_{\sigma}$ and $E_{\sigma}^{\prime}$ are sequentially complete and $E$ has a weak Schauder basis $\left(e_{i}\right)_{i \geq 1}$, then $\widetilde{\sigma}$ is the smallest compatible topology on $E$ for which $\left(e_{i}\right)_{i}$ is an equicontinuous Schauder basis.

Proof. $\quad E_{\sigma}^{\prime}$ is sequentially complete, then $\Delta=\Lambda^{\beta}$ (lemma 3.1) and so $\left(\Lambda, \widetilde{\sigma}\left(\Lambda, \Lambda^{\beta}\right)\right)^{\prime}=\Delta, \operatorname{since}\left(\Lambda, \widetilde{\sigma}\left(\Lambda, \Lambda^{\beta}\right)\right)^{\prime}=\Lambda^{\beta}$. Consequently $\left(E, \widetilde{\sigma}\left(E, E^{\prime}\right)\right)^{\prime}$ $=E^{\prime}$ (example 1. 3) and the result follows from propositions 15 and [10], proposition 1.2, p. 278 .

\section{Compatibility of the $\widetilde{\tau}$-topology}

We establish the compatibility and the completeness of $\widetilde{\tau}$ by distinguishing the three cases: $K$ is local, $K$ is spherically complete and $K$ is non spherically complete.

\section{a. $K$ is local}

Lemma 5. Let $E$ be a topological vector space with an equicontinuous Schauder basis $\left(e_{i}\right)_{i}$; then for every $A \subset E$ the following are equivalent:

a. $A$ is precompact;

b. (i). for all $i \geq 1, p_{i}(A)$ is precompact and (ii). $\left(\sum_{i=1}^{n} p_{i}\right)_{n}$ converges uniformly on $A$. Where the $p_{n}$ are defined in $\S .2$.

Proof. $\quad \mathrm{a} \Longrightarrow \mathrm{b}$ For every $i \geq 1 p_{i}(A)$ is precompact ( $p_{i}$ is continuous). On the other hand, the sequence of linear mappings $\left(\sum_{i=1}^{n} p_{i}\right)_{n}$ is equicontinuous and converging pointwise to a mapping $i d_{E}$ and $A$ is compactoid, then $\left(\sum_{i=1}^{n} p_{i}\right)_{n}$ converges uniformly on $A(\S .3$, lemma 3$)$.

$\mathrm{b} \Longrightarrow$ a Let $U$ be a zero-neighbourhood, then there exists $V$ a neighbourhood of zero and $n_{0} \in \mathbb{N}^{*}$ such that $V+V \subset U$ and $\sum_{n_{0}<i} p_{i}(a) \in V$ , for all $a \in A$. On the other hand $B=\sum_{i=1}^{n_{0}} p_{i}(A)$ is precompact, then 
there exist $b_{1}, b_{2}, \ldots, b_{p} \in E$ such that $B \subset \bigcup_{i=1}^{p}\left(b_{i}+V\right)$. Then $A \subset B+V \subset$ $\bigcup_{i=1}^{p}\left(b_{i}+V\right)+V \subset \bigcup_{i=1}^{p}\left(b_{i}+U\right)$.

Lemma 6. For every $n \geq 1$, the mapping $p_{n}: E_{\sigma}^{\prime} \longrightarrow E_{\widetilde{\sigma}}^{\prime}, \sum_{i=1}^{\infty} \mu_{i} f_{i} \longmapsto$ $\mu_{n} f_{n}$, is continuous; where $\left(f_{i}\right)_{i}$ is the weak Schauder basis asssociated to $\left(e_{i}\right)_{i}$.

Proof. Let $n \geq 1$ and $x \in E$, then for every $f=\sum_{i=1}^{\infty} \mu_{i} f_{i}=\sum_{i=1}^{\infty}\left\langle e_{i}, f\right\rangle f_{i}$ we have: $\widetilde{p}_{x}\left(p_{n}(f)\right)=\sup _{m}\left|\left\langle x, T_{m}\left(p_{n}(f)\right)\right\rangle\right|=\left|\left\langle x, p_{n}(f)\right\rangle\right|=\left|\left\langle x, f_{n}\right\rangle\right|\left|\left\langle x_{n}, f\right\rangle\right|$.

Take $y=\left\langle x, f_{n}\right\rangle x_{n}$, then $y \in E$ and we have $p_{y}(f)=\widetilde{p}_{x}\left(p_{n}(f)\right)$.

Remark 7. Lemma 6 is true for every $K$.

Lemma 7. Let $A \in \mathcal{A}$, then the statements a. and $\mathbf{b}$. are equivalente

a. $A$ is precompact in $E_{\widetilde{\sigma}}^{\prime}$; 0.

b. (i). $A$ is precompact in $E_{\sigma}^{\prime}$ and (ii). for all $x \in E, \lim _{n} \widetilde{p}_{A}\left(x-S_{n}(x)\right)=$

Proof. We consider $A \in \mathcal{A}$ such that $A$ is precompact in $E_{\widetilde{\sigma}}^{\prime}$, then $A$ is precompact in $E_{\sigma}^{\prime}(\sigma \leq \widetilde{\sigma})$. On the other hand, for every $x \in E$, we have :

$$
\widetilde{p}_{A}\left(x-S_{n}(x)\right)=\sup _{k \in \mathbb{N}} p_{A}\left(S_{k}\left(x-S_{n}(x)\right)\right)=\sup _{f \in A} \widetilde{p}_{x}\left(f-T_{n}(f)\right) .
$$

Since $\left(e_{i}\right)_{i}$ is a Schauder basis of $(E, \tau)$, the sequence $\left(f_{i}\right)_{i}$ is an equicontinuous Schauder basis of $E_{\tilde{\sigma}}^{\prime}$ [9], lemma 3, p. 402 and [10], proposition 1.2, p. 278.

Furthermore $A$ is precompact in $E_{\tilde{\sigma}}^{\prime}$, so $\left(T_{n}\right)_{n}$ converges to $i d_{E^{\prime}}$ uniformly on $A$ in $E_{\widetilde{\sigma}}^{\prime}(\S .3$, lemma 3$)$; then $\lim _{n \rightarrow \infty} \sup _{f \in A} \widetilde{p}_{x}\left(f-T_{n}(f)\right)=0$ for all $x \in E$, and so $\lim _{n \rightarrow \infty} \widetilde{p}_{A}\left(x-S_{n}(x)\right)=0$.

Conversely $A$ is precompact in $E_{\sigma}^{\prime} \Longrightarrow$ for all $i \geq 1 p_{i}(A)$ is precompact in $E_{\tilde{\sigma}}^{\prime}$ (lemma 6). On the other hand we have for all $x \in E$ and all $n \geq 1 \widetilde{p}_{A}\left(x-S_{n}(x)\right)=\sup _{f \in A} \widetilde{p}_{x}\left(f-T_{n}(f)\right)=\sup _{f \in A} \widetilde{p}_{x}\left(f-\sum_{i=1}^{n} p_{i}(f)\right)$. So $\lim _{n \rightarrow \infty} \widetilde{p}_{x}\left(f-\sum_{i=1}^{n} p_{i}(f)\right)=0$ for all $x \in E$, this means that $\left(\sum_{i=1}^{n} p_{i}\right)_{n}$ 
converges uniformly to $i d_{E^{\prime}}$ on $A$ in $E_{\widetilde{\sigma}}^{\prime}$; then by lemma $5, A$ is precompact in $E_{\tilde{\sigma}}^{\prime}$.

Lemma 8. If $E^{\prime}$ is $\sigma\left(E^{\prime}, E\right)$-sequentially complete, then for every $A \in \mathcal{A}$, the following are equivalent:

a. $A$ is $\widetilde{\sigma}$-relatively compact;

b. (i). $A$ is relatively compact in $E_{\sigma}^{\prime}$ and (ii). for all $x \in E$, $\lim _{n \rightarrow \infty} \widetilde{p}_{A}\left(x-S_{n}(x)\right)=0$.

Proof. Suppose that $A$ is $\widetilde{\sigma}$-relatively compact in $E_{\widetilde{\sigma}}^{\prime} ; \bar{A}^{\widetilde{\sigma}}$ is compact in $E_{\sigma}^{\prime}(\sigma \leq \tilde{\sigma})$. Since $A \subset \bar{A}^{\widetilde{\sigma}}$ and $\bar{A}^{\widetilde{\sigma}}$ is closed in $E_{\sigma}^{\prime}$ then $\bar{A}^{\sigma} \subset \bar{A}^{\widetilde{\sigma}}$ and so $\bar{A}^{\sigma}$ is compact in $E_{\sigma}^{\prime}$. Furthermore by lemma 7 we have (ii).

Conversely, take $A$ such that (i) and (ii) of $\mathbf{b}$ holds, then $A$ and so $\bar{A}^{\widetilde{\sigma}}$ are precompacts in $E_{\tilde{\sigma}}^{\prime}$ (lemma 7). Consequently $\bar{A}^{\widetilde{\sigma}}$ is compact in $E_{\tilde{\sigma}}^{\prime}\left(E_{\tilde{\sigma}}^{\prime}\right.$ is complete: proposition 15.ii).

Proposition 18. Let $A \in \mathcal{A}$.

1. $\widetilde{A}$ is $\widetilde{\sigma}\left(E^{\prime}, E\right)$ - precompact;

2. If $E^{\prime}$ is $\sigma\left(E^{\prime}, E\right)$ - sequentially complete, then

i. $\widetilde{A}$ is $\widetilde{\sigma}\left(E^{\prime}, E\right)$ - relatively compact;

ii. $\Gamma(\widetilde{A})$ is $\sigma\left(E^{\prime}, E\right)$ - relatively compact.

Proof. $\quad$ Let $A \in \mathcal{A}$.

1. $\widetilde{A}$ is $\sigma\left(E^{\prime}, E\right)$ - bounded [10], lemma 1.2 , p. 277, then it is $\sigma\left(E^{\prime}, E\right)$ - relatively compact ([2], proposition 2.3 , p. 223) and so $\widetilde{A}$ is precompact in $\left(E^{\prime}, \sigma\left(E^{\prime}, E\right)\right)$. On the other hand for every $x \in E$ $\lim _{n \rightarrow \infty} \widetilde{p}_{A}\left(x-S_{n}(x)\right)=0\left(\left(e_{i}\right)_{i}\right.$ is a Schauder basis of $\left.(E, \widetilde{\tau})\right)$. Therefore, by lemma 7 and remarks $2, \widetilde{A}$ is precompact in $E_{\widetilde{\sigma}}^{\prime}$.

2. $\widetilde{A}$ is $\sigma\left(E^{\prime}, E\right)$-relatively compact and $\lim _{n \rightarrow \infty} \widetilde{p}_{A}\left(x-S_{n}(x)\right)=0$ for every $x \in E$, then $\widetilde{A}$ is $\widetilde{\sigma}\left(E^{\prime}, E\right)$ - relatively compact (lemma 8).

3. $\widetilde{A}$ is $\widetilde{\sigma}\left(E^{\prime}, E\right)$ - relatively compact, so $B=\overline{\Gamma(\widetilde{A})}^{\widetilde{\sigma}}$ is $\widetilde{\sigma}\left(E^{\prime}, E\right)$ - compact because $B$ is a closed in a complete space $E_{\tilde{\sigma}}^{\prime}$ (proposition 15 and [30], p. 26). Hence $\Gamma(\widetilde{A})$ is $\sigma\left(E^{\prime}, E\right)$ - relatively compact in $E_{\sigma}^{\prime}$ (lemma 8). 
Proposition 19. If $(E, \tau)$ has a Schauder basis and $E^{\prime}$ is $\sigma\left(E^{\prime}, E\right)$ sequentially complete, then $\widetilde{\tau}$ is compatible with the duality $\left\langle E, E^{\prime}\right\rangle$.

Proof. We have $\sigma \leq \widetilde{\tau}$. On the other hand, $\widetilde{\tau}$ is generated by the family $\left(\overline{\Gamma(\widetilde{A})}^{\sigma\left(E^{\prime}, E\right)}\right)_{A \in \mathcal{A}}\left([2], \S .3\right.$, proposition 3.4, p. 228) and $\overline{\Gamma(\widetilde{A})}^{\sigma\left(E^{\prime}, E\right)}$ is $\sigma\left(E^{\prime}, E\right)$ - compact for every $A \in \mathcal{A}$, so $\widetilde{\tau} \leq \tau_{m}$, where $\tau_{m}$ is the Mackey topology on $E$.

\section{b. $K$ is spherically complete}

Lemma 9. Let $E$ be a topological $K$-vector space with an equicontinuous Schauder basis $\left(e_{i}\right)_{i}$; then for every $A \subset E$ the statements $\mathbf{a}$ and $\mathbf{b}$ are equivalente

a. $A$ is compactoid;

b. (i). for all $i \geq 1 \quad p_{i}(A)$ is compactoid and (ii). $\left(\sum_{i=1}^{n} p_{i}\right)_{n}$ converges uniformly on $A$.

Proof. Suppose that $A$ is compactoid; then for every $i \geq 1 p_{i}(A)$ is compactoid. On the other hand, $\left(e_{i}\right)_{i}$ is an equicontinuous Schauder basis, so $\left(\sum_{i=1}^{n} p_{i}\right)_{n}$ converges pointwise to the mapping $i d_{E}$; since $A$ is compactoid, this convergence is uniform on $A$ (§. 3, lemma 3$)$.

Conversely let $U$ and $V$ are two zero-neigbourhoods such that $V+V \subset$ $U$, then the convergence of $\left(\sum_{i=1}^{n} p_{i}\right)_{n}$ on $A$ implies the existence of $n_{0} \in \mathbb{N}$ such that $\sum_{i=n_{0}+1}^{\infty} p_{i}(x) \in V$ for all $x \in A$. On the other hand, (i) of lemma induces the existance of $x_{1}, \ldots, x_{n} \in E$ such that $\sum_{i=1}^{n_{0}} p_{i}(A) \subset V+\Gamma(B)$, where $B=\left\{x_{1}, \ldots, x_{n}\right\}\left(\sum_{i=1}^{n_{0}} p_{i}(A)\right.$ is compactoid $)$.

Then for every $x \in A, x=\sum_{i=1}^{n_{0}} \lambda_{i} e_{i}+\sum_{i=n_{0}+1}^{\infty} \lambda_{i} e_{i}$ 
so $A \subset U+\Gamma(B)$.

$$
=\sum_{i=1}^{n_{0}} p_{i}(x)+\sum_{i=n_{0}+1}^{\infty} p_{i}(x) \in V+\Gamma(B)+V
$$

Lemma 10. Let $A \in \mathcal{A}$, then the following are equivalent

a. $A$ is compactoid in $E_{\tilde{\sigma}}^{\prime}$;

b. (i). $A$ is compactoid in $E_{\sigma}^{\prime}$ and (ii). for all $x \in E \lim _{n \rightarrow \infty} \widetilde{p}_{A}\left(x-S_{n}(x)\right)=$ 0 .

Proof. $\quad$ Same proof as for lemma 7 using lemma 9 and remark 7.

Proposition 20. Let $\tau$ be a polar topology of $\mathcal{A}$ - convergence on $E$ and $\left(e_{i}\right)_{i}$ be a Schauder basis of $(E, \tau)$, then for every $A \in \mathcal{A}$

i. $\widetilde{A}$ is $\widetilde{\sigma}$-compactoid;

ii. If $E^{\prime}$ is $\sigma\left(E^{\prime}, E\right)$ - sequentially complete then

a. $\widetilde{A}$ is $\widetilde{\sigma}-$ relatively-c-compact;

b. $\Gamma(\widetilde{A})$ is $\sigma$ - relatively-c-compact.

Proof. $\quad$ i. Let $A \in \mathcal{A}$, then $\widetilde{A}$ is $\sigma$-bounded [10], lemma 1.2, p. 277 and, since $K$ is spherically complete, $\widetilde{A}$ is compactoid in $E_{\sigma}^{\prime}$ ([31], proposition 18.ii, p. 145). On the other hand for all $x \in E \lim _{n \rightarrow \infty} \widetilde{p}_{A}\left(x-S_{n}(x)\right)=0$ since $\left(e_{i}\right)_{i}$ is a Schauder basis of $(E, \widetilde{\tau})$. Then $\widetilde{A}$ is copmactoid in $E_{\widetilde{\sigma}}^{\prime}$ (lemma $10)$.

ii. Let $A \in \mathcal{A}$; then

a. $\widetilde{\widetilde{A}}^{\widetilde{\sigma}}$ is compactoid in $E_{\tilde{\sigma}}^{\prime}$ (by i) $\Longrightarrow E_{\tilde{\sigma}}^{\prime}$ is complete, because $E^{\prime}$ is $\sigma\left(E^{\prime}, E\right)$ - sequentially complete (proposition 15), then $\widetilde{\widetilde{A}}^{\widetilde{\sigma}}$ is also complete and so it is c-compact in $E_{\widetilde{\sigma}}^{\prime}$ [31], theorem 9, p. 141.

b. $B=\overline{\Gamma(\widetilde{A})}^{\widetilde{\sigma}}$ is c-compact in $E_{\widetilde{\sigma}}^{\prime}$, then it is $\sigma\left(E^{\prime}, E\right)-c$-compact and $\sigma\left(E^{\prime}, E\right)$-closed $(\sigma \leq \widetilde{\sigma})$, therefore $\overline{\Gamma(\widetilde{A})^{\sigma}}\left(E^{\prime}, E\right)$ is $\sigma\left(E^{\prime}, E\right)-c-$ compact.

Proposition 21. If $(E, \tau)$ has a Schauder basis and $E^{\prime}$ is $\sigma\left(E^{\prime}, E\right)-$ sequentially complete, then $\widetilde{\tau}$ is compatible with the duality $\left\langle E, E^{\prime}\right\rangle$. 
Proof. The topology $\widetilde{\tau}$ is a polar topology of $\mathcal{B}$ - convergence; where $\mathcal{B}=\left(\overline{\Gamma(\widetilde{A})}^{\sigma}\left(E^{\prime}, E\right)\right)_{A \in \mathcal{A}}$ and $\mathcal{A}$ is the family which defines the topology $\tau ;$ for every $A \in \mathcal{A},{\overline{\Gamma(\widetilde{A})^{\sigma}}}^{\sigma}\left(E^{\prime}, E\right)$ is $\sigma\left(E^{\prime}, E\right)$-c-compact in $E_{\sigma}^{\prime}$ (proposition 20 ), then $\widetilde{\tau}$ is compatible with the duality $\left\langle E, E^{\prime}\right\rangle[2]$, theorem 4.4, p. 234.

\section{c. $K$ is not spherically complete}

$K$ is not spherically complete $\Longrightarrow K$ is dense $\Longrightarrow$ For every absolutely $K-$ convex $A$ in $E^{\prime}, K_{c}(A)=\bigcap_{|\lambda| \succ 1} \lambda A \Longrightarrow$ for all $|\mu| \succ 1 \mu K_{c}(A)=$ $K_{c}(\mu A)$. Then we have the following proposition :

Proposition 22. If $(E, \tau)$ has a Schauder basis, then $\widetilde{\tau}$ is compatible.

Proof. Let $\mathcal{A}$ be a family which defines the topology $\tau$, such that for all $A \in \mathcal{A} A$ is absolutely $K$ - convex; then $K_{c}\left(\widetilde{\widetilde{A}}^{\sigma\left(E^{\prime}, E\right)}\right)^{\circ}=(\widetilde{A})^{\circ}$ for all $\mathrm{A} \in \mathcal{A}[2]$, corollary 4.3, p. 233, so if we take $\beta=\left(K_{c}\left(\overline{\widetilde{A}}^{\sigma\left(E^{\prime}, E\right)}\right)\right)_{A \in \mathcal{A}}$, then $\beta$ verify the conditions (a), (b) and (c) of 8 . Therefore $\widetilde{\tau}$ is a polar topology of $\beta$ - convergence and its elements are $E$ - closed. Then $\widetilde{\tau}$ is compatible [2], theorem 4.3, p. 233.

\section{Completeness of the topology $\widetilde{\tau}$}

Proposition 23. Let $(E, \tau)$ be a locally $K$-convex space and $\left(e_{i}\right)_{i}$ be a Schauder basis of $(E, \tau)$. If $E$ and $E^{\prime}$ are weakly-sequentially complete, then $(E, \widetilde{\tau})$ is complete.

Proof. The space $\left(E, \widetilde{\sigma}\left(E, E^{\prime}\right)\right)$ is complete (proposition 15), then by remarks 2 and $([2]$, theorem 3.2 , p. 230$)(E, \widetilde{\tau})$ is complete.

The following theorem is a consequence for previous results

Theorem 6. Let $(E, \tau)$ be a locally $K$ - convex space with a Schauder basis $\left(e_{i}\right)_{i}$ such that $E_{\sigma}$ and $E_{\sigma}^{\prime}$ are sequentially complete, then $\widetilde{\tau}$ is complete and it is the coarsest compatible topology on $E$ finer than $\tau$ for which $\left(e_{i}\right)_{i}$ is an equicontinuous Schauder basis. 


\section{The weak basis Problem}

Throughout this section we shall assume that $(E, \tau)$ has a weak Schauder basis $\left(e_{i}\right)_{i}$ and the spaces $E_{\sigma}^{\prime}$ and $E_{\sigma}$ are sequentially complete. We then characterize the finest $\left(E, E^{\prime}\right)$-compatible topology on $E$ for which $\left(e_{i}\right)_{i}$ is a Schauder basis; according to theorem $6,\left(e_{i}\right)_{i}$ is equicontinuous for that topology. We shall distinguish three cases: $K$ is local, $K$ is spherically complete or $K$ is not spherically complete.

a. $K$ is local

Let $\mathcal{B}=\left\{B \subset E^{\prime} / B=\widetilde{B}\right.$ and $B$ is $\widetilde{\sigma}-$ precompact $\} ;$ it is obviously that $\mathcal{B}$ is not empty and verifies the properties $(a),(b)$ and $(c)$ of 8 . Let $\mathcal{U}$ be the polar topology of $\mathcal{B}$-convergence on $E$; we have the following propositions

Proposition 24. $\mathcal{U}$ is compatible with the duality $\left\langle E, E^{\prime}\right\rangle$ and $\left(e_{i}\right)_{i}$ is an equicontinuous Schauder basis of $(E, \mathcal{U})$.

Proof. $E^{\prime}$ is $\sigma\left(E^{\prime}, E\right)$ - sequentially complete $\Longrightarrow$ for all $B \in \mathcal{B}$, $\overline{\Gamma(B)}^{\sigma}\left(E^{\prime}, E\right)$ is $\sigma\left(E^{\prime}, E\right)-$ compact (proposition 18) $\Longrightarrow \mathcal{U}$ is compatible with the duality $\left\langle E, E^{\prime}\right\rangle[2]$, theorem 4.5, p. 235.

We'll prove that $\left(e_{i}\right)_{i}$ is a Schauder basis of $(E, \mathcal{U}) .\left(e_{i}\right)_{i}$ is a weak Schauder basis $\Longrightarrow\left(f_{i}\right)_{i}$ is a Schauder basis of $\left(E^{\prime}, \sigma\left(E^{\prime}, E\right)\right)$, then $\left(f_{i}\right)_{i \geq 1}$ is an equicontinuous Schauder basis of $\left(E^{\prime}, \widetilde{\sigma}\left(E^{\prime}, E\right)\right)$; therefore $\left(T_{n}\right)_{n}$ is equicontinuous in $\left(E^{\prime}, \widetilde{\sigma}\left(E^{\prime}, E\right)\right)$ and converges pointwise to the mapping $i d_{E^{\prime}}$, then the convergence is uniformly on every $B \in \mathcal{B}$, this means that for all $x \in E \limsup _{n} \widetilde{p}_{f \in B}\left(f-\sum_{i=1}^{n} p_{i}(f)\right)=0$.

For every $x \in E$ and for every $B \in \mathcal{B}$ we have $\sup _{f \in B} \widetilde{p}_{x}\left(f-\sum_{i=1}^{n} p_{i}(f)\right)=$ $\widetilde{p}_{B}\left(x-S_{n}(x)\right)$ (lemma 7$)$. Then $\lim _{n} \widetilde{p}_{B}\left(x-S_{n}(x)\right)=0$ for all $x \in E$ and for all $B \in \mathcal{B}$, and so $\left(S_{n}(x)\right)_{n}$ converges to $x$ in $(E, \mathcal{U})$ for every $x \in E$.

Moreover the associated sequence $\left(f_{i}\right)_{i}$ of $\left(e_{i}\right)_{i}$ verifies for all $i \geq 1$ $f_{i} \in(E, \mathcal{U})^{\prime}$ ( $U$ is compatible).

Proposition 25. $\mathcal{U}$ is the finest compatible topology on $E$ for which $\left(e_{i}\right)_{i}$ is a Schauder basis. 
Proof. Let $\tau$ be a compatible topology on $E$ such that $\left(e_{i}\right)_{i}$ be a Schauder basis of $(E, \tau)$. Then $B=\overline{\Gamma(\widetilde{A})}^{\sigma\left(E^{\prime}, E\right)}$ is a $\sigma\left(E^{\prime}, E\right)-$ compact of $E^{\prime}$ (proposition 18) and since $B=\widetilde{B}, B^{\circ}$ is a zero-neighbourhood in $(E, \mathcal{U})$; now $B^{\circ}=(\widetilde{A})^{\circ}$, for every $A \in \mathcal{A}$, where $\mathcal{A}$ is a family which defines the topology $\tau$, then $\widetilde{\tau} \leq \mathcal{U}$ and so $\tau \leq \mathcal{U}$.

Proposition 26. A weak Schauder basis $\left(e_{i}\right)_{i}$ is a Schauder basis for a polar compatible topology on $E$ if, and only if, for every $A \in \mathcal{A}, \widetilde{A}$ is $\widetilde{\sigma}\left(E^{\prime}, E\right)$ - relatively compact.

Proof. $\quad \Longrightarrow$ B By proposition 18 .

$\Longleftarrow]$ Suppose that for every $A \in \mathcal{A}, \widetilde{A}$ is $\widetilde{\sigma}\left(E^{\prime}, E\right)$-relatively compact, then $\overline{\Gamma(\widetilde{A})}{ }^{\sigma}\left(E^{\prime}, E\right)$ is $\sigma\left(E^{\prime}, E\right)-$ compact (proposition 18), so $\tau \leq U$ and $\left(e_{i}\right)_{i}$ is a Schauder basis of $(E, \tau)$.

Corollary 4. A weak Schauder basis $\left(e_{i}\right)_{i}$ is a Schauder basis for a polar compatible topology $\tau$ on $E$ if, and only if, for every $A \in \mathcal{A}, \widetilde{A}$ is $\widetilde{\sigma}\left(E^{\prime}, E\right)-$ relatively compact, where $\mathcal{A}$ is a family that define the topology $\tau$.

Proof. $\quad \tau_{m}$ is compatible, then it suffices to use the proposition 26 .

Remark 8. If $\tau$ is a polar topology of $\mathcal{A}$ - convergence on $E$ having a weak Schauder basis $\left(e_{i}\right)_{i}$, then for every $A \in \mathcal{A}, \widetilde{A}$ is $\widetilde{\sigma}\left(E^{\prime}, E\right)$ - relatively compact.

Proof. $E$ is an $O P$ - space, so $\left(e_{i}\right)_{i}$ is a Schauder basis of $(E, \tau)$, then according to proposition 18 we have the conclusion.

\section{b. $K$ is spherically complete}

Let $\mathcal{N}=\left\{N \subset E^{\prime} / N=\widetilde{N}\right.$ and $N$ is $\widetilde{\sigma}\left(E^{\prime}, E\right)$ - compactoid $\}$; it is obviously that $\mathcal{N}$ is not empty and verifies the properties $(a),(b)$ and $(c)$ of 8. Let $\mathcal{V}$ the polar topology of $\mathcal{N}$-convergence on $E$, then we have the following propositions

Proposition 27. The topology $\mathcal{V}$ is compatible with the duality $\left\langle E, E^{\prime}\right\rangle$ and $\left(e_{i}\right)_{i}$ is an equicontinuous Schauder basis of $(E, \mathcal{V})$. 
Proof. Same proof as for proposition 24 using the proposition 20 and [2], theorem 4.4, p. 234.

Proposition 28. $\mathcal{V}$ is the finest compatible topology on $E$ for which $\left(e_{i}\right)_{i}$ is a Schauder basis.

Proof. Same proof as for proposition 25 using the proposition 20.

Proposition 29. A weak Schauder basis $\left(e_{i}\right)_{i}$ is a Schauder basis for a polar compatible topology $\tau$ on $E$ if, and only if, for every $A \in \mathcal{A}, \widetilde{A}$ is $\tilde{\sigma}\left(E^{\prime}, E\right)$-relatively c-compact, where $\mathcal{A}$ is a family that defines the topology $\tau$.

Proof. Same proof as for proposition 26 using the proposition 20.

Remark 9. if $\tau$ is a polar topology of $\mathcal{A}$-convergence on $E$ that having a weak Schauder basis $\left(e_{i}\right)_{i}$, then for every $A \in \mathcal{A}, \widetilde{A}$ is $\widetilde{\sigma}\left(E^{\prime}, E\right)$ - relatively c-compact.

Corollary 5. A weak Schauder basis $\left(e_{i}\right)_{i}$ is a Schauder basis for a topology $\tau_{c}$ on $E$ if, and only if, for every absolutely $K$-convex, $\sigma\left(E^{\prime}, E\right)$-bounded and $\sigma\left(E^{\prime}, E\right)$-c-compact $A$ of $E^{\prime}, \widetilde{A}$ is $\widetilde{\sigma}\left(E^{\prime}, E\right)$-relatively c-compact.

Proof. It is sufficient to take $\tau=\tau_{c}$ in proposition 29 .

c. $K$ is not spherically complete

Let $\mathcal{M}$ the family of all $M \subset E^{\prime}$ such that $M=\widetilde{M}, M$ is $\sigma\left(E^{\prime}, E\right)-$ bounded, $E-$ closed and $\left(T_{n}\right)_{n}$ converges uniformly on $M$ in $E_{\tilde{\sigma}}^{\prime}$, where $E_{\widetilde{\sigma}}^{\prime}=\left(E^{\prime}, \widetilde{\sigma}\left(E^{\prime}, E\right)\right)$. Let $\vartheta$ be the polar topology of $\mathcal{M}$-convergence.

Theorem 7. $\vartheta$ is the finest compatible topology on $E$ for which $\left(e_{i}\right)_{i}$ is an equicontinuous Schauder basis.

Proof. $\quad \vartheta$ is compatible [2], theorem 4.3, p. 233.

Let $M \in \mathcal{M}$, then $\left(T_{n}\right)_{n}$ converges uniformly on $M$ in $E_{\tilde{\sigma}}^{\prime} \Longrightarrow \lim _{n} \sup _{f \in M}$ $\widetilde{p}_{x}\left(f-T_{n}(f)\right)=0$, so $\lim _{n} p_{M}\left(x-S_{n}(x)\right)=0$. Then $\left(e_{i}\right)_{i}$ is a Schauder basis of $\vartheta$.

Let $\tau$ be a polar and compatible topology of $\mathcal{A}$ - convergence such that $\left(e_{i}\right)_{i}$ be an equicontinuous Schauder basis, then $\tau=\widetilde{\tau}$. Therefore 
$\mathcal{A}=\left\{A \subset E^{\prime} / A=\widetilde{A}\right.$ and $A$ is $\sigma\left(E^{\prime}, E\right)$ - bounded and $E$-closed $\}$.

Let $A \in \mathcal{A}$, so for every $x \in E \lim _{n} p_{A}\left(x-S_{n}(x)\right)=0$, then $\lim _{n}$ $\sup _{f \in A} \tilde{p}_{x}\left(f-T_{n}(f)\right)=0$, therefore $\left(T_{n}\right)_{n}$ converges uniformly on $A$ in $E_{\tilde{\sigma}}^{\prime}$. Then $\tau \leq \vartheta$.

Theorem 8. Let $\tau$ be a polar topology of $\mathcal{A}$ - convergence on $E$ such that $E_{\sigma}$ and $E_{\sigma}^{\prime}$ are sequentially complete and $\left(e_{i}\right)_{i}$ be a weak Schauder basis of $E$, then $\left(e_{i}\right)_{i}$ is a Schauder basis of $(E, \tau)$ if, and only if, for all $A \in \mathcal{A}$ the sequence $\left(T_{n}\right)_{n}$ converges uniformly on $A$ in $E_{\tilde{\sigma}}^{\prime}$.

Proof. If $\left(e_{i}\right)_{i}$ is a Schauder basis of $(E, \tau)$, then $\left(e_{i}\right)_{i}$ is an equicontinuous Schauder basis of $\widetilde{\tau}$; therefore for all $A \in \mathcal{A}$ the sequence $\left(T_{n}\right)_{n \geq 1}$ converges uniformly on $\widetilde{A}$ in $E_{\widetilde{\sigma}}^{\prime}$, so for all $A \in \mathcal{A},\left(T_{n}\right)_{n}$ converges uniformly on $A$ in $E_{\tilde{\sigma}}^{\prime}$.

Conversely, let $A \in \mathcal{A}$, then for all $x \in E \quad \widetilde{p}_{A}\left(x-S_{n}(x)\right)=\sup _{f \in A}$ $\widetilde{p}_{x}\left(f-T_{n}(f)\right)$.

But $\lim _{n} \sup _{f \in A} \widetilde{p}_{x}\left(f-T_{n}(f)\right)=0$, then $\lim _{n} \widetilde{p}_{A}\left(x-S_{n}(x)\right)=0$ and so $\lim _{n} p_{A}\left(x-S_{n}(x)\right)=0$.

Theorem 9. Under the conditions of theorem 8,E is an $O P$ - space if, and only if, for all $A \in \mathcal{A}$ the sequence $\left(T_{n}\right)_{n}$ converges uniformly on $A$ in $E_{\widetilde{\sigma}}^{\prime}$.

Proof. ([21], proposition 1) and theorem 8.

Proposition 30. Let $\tau$ be a polar topology of $\mathcal{A}$ - convergence on $E$ and $\left(e_{i}\right)_{i}$ be a weak Schauder basis of $E$, then if every $\tilde{\sigma}-$ equicontinuous sequence of $E^{\prime}$ that converging pointwise to zero converges uniformly on every $A \in \mathcal{A}$ in $E_{\tilde{\sigma}}^{\prime}$, then $\left(e_{i}\right)_{i}$ is a Schauder basis of $(E, \tau)$.

Proof. $\quad\left(f_{i}\right)_{i}$ is an equicontinuous Schauder basis of $\left(E^{\prime}, \widetilde{\sigma}\left(E^{\prime}, E\right)\right)=$ $E_{\tilde{\sigma}}^{\prime} \Longrightarrow\left(T_{n}\right)_{n}$ is an equicontinuous sequence of $E_{\tilde{\sigma}}^{\prime} \Longrightarrow$ the sequence $\left(i d_{E^{\prime}}-T_{n}\right)_{n}$ is pointwise converging to zero in $E_{\widetilde{\sigma}}^{\prime}$ and this convergence is uniformly on every $A \in \mathcal{A}$ in $E_{\widetilde{\sigma}}^{\prime} \Longrightarrow \lim _{n} \sup _{f \in A} \widetilde{p}_{x}\left(f-T_{n}(f)\right)=0$ for all $x \in E \Longrightarrow \lim _{n} \widetilde{p}_{A}\left(x-S_{n}(x)\right)=0$ for all $x \in E \Longrightarrow \lim _{n} p_{A}\left(x-S_{n}(x)\right)=0$ for all $x \in \stackrel{n}{E}$. 


\section{Application to barrelled spaces and G-spaces}

\section{Barrelled spaces}

\section{a. $K$ is spherically complete}

Proposition 31. If $(E, \tau)$ is a barrelled locally $K$ - convexe space which is $\sigma\left(E, E^{\prime}\right)$ - sequentially complete and having a weak Schauder basis, then $(E, \tau)$ is complete and every weak Schauder basis is an equicontinuous Schauder basis of $(E, \tau)$.

Lemma 11. If $(E, \tau)$ is barrelled and having a Schauder basis then $\tau=\widetilde{\tau}$.

Proof. Let $A \in \mathcal{A}$, where $\mathcal{A}$ is a family that defines the topology $\tau$; $(\widetilde{A})^{\circ}$ is a barrel, so it is a zero-neighbourhood in $(E, \tau)$, then $\widetilde{\tau} \leq \tau$.

Proof of proposition Let $\left(e_{i}\right)_{i}$ be a weak Schauder basis of $E$, then $\left(e_{i}\right)_{i}$ is a Schauder basis of $(E, \tau)(E$ is an $O P-$ space $) \Longrightarrow\left(e_{i}\right)_{i}$ is an equicontinuous Schauder basis of $\widetilde{\tau} \Longrightarrow\left(e_{i}\right)_{i}$ is an equicontinuous Schauder basis of $(E, \tau)(\tau=\widetilde{\tau})$.

b. $\mathbf{K}$ is not spherically complete

Proposition 32. Every weak Schauder basis in a polarly barrelled polar locally $K$-convex space is an orthogonal basic sequence.

Proof. ([21], corollary 6, p. 155).

Proposition 33. Let $E$ be a Banach space with a weak Schauder basis; then $E$ is a polar space if and only if, every weak Schauder basis in $E$ is a basic sequence.

Proof. For the sufficient condition, one only has to use theorem $3.2(\alpha)$ $\Longrightarrow(\beta)$ of $[28]$. The necessary condition is a particular case of proposition 32 .

\section{G-spaces}

Proposition 34. If $(E, \tau)$ is a weakly-sequentially complete $G$-space that having a Schauder basis, then $(E, \tau)$ is complete and this basis is equicontinuous.

Proof. By theorem 6 . 
Proposition 35. If $(E, \tau)$ is a $G$-space with a Schauder basis $\left(e_{i}\right)_{i}$, there is no strictly finer locally $K$-convex topology on $E$ for which $\left(e_{i}\right)_{i}$ is still a Schauder basis.

Proof. By proposition 19 if $K$ is local, proposition 21 if $K$ is spherically complete and by proposition 22 if $K$ is not spherically complete.

Proposition 36. Suppose that $K$ is spherically complete; if $(E, \tau)$ is a weakly-sequentially complete $G$-space that having a weak Schauder basis $\left(e_{i}\right)_{i}$, then $(E, \tau)$ is complete and $\left(e_{i}\right)_{i}$ is an equicontinuous Schauder basis of $(E, \tau)$.

Proof. Let $\left(e_{i}\right)_{i}$ be a weak Schauder basis of $E$, then $E$ is an $O P$-space $\Longrightarrow\left(e_{i}\right)_{i}$ is a Schauder basis of $(E, \tau)$. So the proposition is an immediate consequence of proposition 34 .

\section{References}

[1] M. G. Arsove, and R. E. Edwards, generalized bases in topological linear spaces. Studia math.19, pp. 95-113, (1960).

[2] R. Ameziane Hassani and M. Babahmed, Topologies polaires compatibles avec une dualité séparante sur un corps valué nonarchimédien, Proyecciones. Vol. 20, No. 2, pp. 217-241, (2001).

[3] S. Banach, Théorie des opérateurs linéaires, Chelsea, New York (1955).

[4] S. Bennet and J. B. Cooper, Weak basis in F and (LF)-spaces. J. London Math. Soc. 44, pp. 505-508, (1969).

[5] G. Bessaga and A. Pełczynski, Properties of bases in spaces of type $B_{0}$. Prace Math. 3, pp. 123-142, (1959).

[6] N. Bourbaki, Espaces vectoriels topologiques, Chap.1 à 5, Paris, (1981).

[7] N. De Grande-De Kimpe, C-compactness in locally $K$-convex spaces, Indag. Math. 33, pp. 176-180, (1971).

[8] N. De Grande-De Kimpe, Perfect locally $K$-convex sequence spaces, Indag. Math. 33, pp. 471-482, (1971). 
[9] N. De Grande-De Kimpe, On the structure of locally $K$-convex spaces with a Schauder basis, Indag. Math. 34, pp. 396-406, (1972).

[10] N. De Grande-De Kimpe, Equicontinuous Schauder basis and compatible locally convex topologies, Proc. Kond. Ned. Akad. V. Wet. A77(3), pp. 276-283 (1973).

[11] N. De Grande-De Kimpe, On a class of locally convex spaces with a Schauder basis, Proc. Kond. Ned.Akad. V. Wet. pp. 307-312, (1976).

[12] N. De Grande-De Kimpe, Structure theorems for locally K-convex spaces. Proc. Kond. Ned. Akad. Wet. 80: pp. 11-22, (1977).

[13] M. De Wilde, Reseaux dans les espaces linéaires a semi-normes. Mem. Soc. R. Liège. (1969).

[14] Dorleyn, M, Beschouwingen over coördinatenruimten, oneindige matrices en determinanten in een niet-archmedisch gewaardeerd lichaam. Thesis, Amsterdam, (1951).

[15] E. Dubinsky, JR. Retherford, Schauder bases in compatible topologies. Stud. Math. 28: pp. 221-226, (1967).

[16] T. A. Efimova, On weak basis in the inductive limits of barrelled normed spaces. Vestnik. Leningrad Uni. Math. Meb. Astronom.119, pp. 21-26, (1981).

[17] K. Floret, Bases in sequentially retractive limits spaces. Proc. Int. Coll.on Nuclear Spaces and Ideals in operators Algebras, Warsaw1969, Studia Math. 38, pp. 221-226, (1970).

[18] D. J. H. Garling, On topological sequence spaces, Proc. Camb. Phil. Soc. 63, pp. 997-1019, (1967).

[19] D. J. H. Garling, The $\beta$-and $\gamma$-duality of sequence spaces, Proc. Camb. Phil. Soc. 63, pp. 963-981, (1967).

[20] N. J. Kalton, On the weak-basis theorem. Compositio Mathematica, Vol. 27, Fasc. 2, pp. 213-215, (1973).

[21] J. Kąkol and T. Gilsdorf, On the weak basis theorems for p-adic locally convex spaces, p-adic functional analysis edited by J. Kạkol, N. De Grande-De Kimpe and C. Perez-Garcia. Marcel Dekker, Ink. New York, (1999). 
[22] J. Ka̧kol, C. Perez-Garcia and W. H. Schikhof, Cardinality and Mackey topologies of non-archimedean Banach and Fréchet spaces. Bull PolAcad Sci Math 44: pp. 131-141, (1996).

[23] G. Köthe, Topologische lineaire Räume; Springer Verlag, (1960).

[24] C. W. McArthur, The weak basis theorem, Colloq. Math. 17, pp. 71-76, (1967).

[25] A. F. Monna, Espaces linéaires à une infinité dé nombrable de coordonnées. Proc. Ned. Akad. V. Wetensch. 53, pp. 1548-1559, (1950).

[26] A. F. Monna, Sur le théorème de Banach-Steinhaus, Proc. Kond. Ned. Akad. V. Wetensch. A66, pp. 121-31 (1963).

[27] J. Orihuela, On the equivalence of weak and Schauder bases, Arch. Math. Vol. 46, pp. 447-452, (1986).

[28] C. Perez-Garcia and W. H. Schikhof, The Orlicz-Pettis property in p-adic analysis, collect. Math. 43, 3, pp. 225-233, (1992).

[29] J. H. Shapiro, On the weak basis theorem in F-Spaces, can. J. Math. Vol. XXVI, No. 6, pp. 1294-1300, (1974).

[30] H. H. Schaefer, Topological vector spaces, Springer-Verlag New-York, herdlberg Berlin, (1971).

[31] W. H. Schikhof, Compact-like sets in non-archimedean fonctional analysis, Proc. of the conférence on p-adic analysis henglehoef, Belgium, pp. 137-147 (1986).

[32] W. H. Schikhof, The continuous linear image of p-adic compactoid. Proc Kon Ned Akad Wet 92: pp. 119-123, (1989).

[33] I. Singer, Weak*-bases in conjugate Banach spaces, Stud. Math. 21, (1961).

[34] T. A. Springer, Une notion de compacité dans la théorie des espaces vectoriels topologiques, Indag. Math. 27, pp. 182-189 (1965).

[35] W. J. Stiles, On properties of subspaces of $l_{p}, 0 \prec p \prec 1$, Trans. mer. Math. Soc. 149, pp. 405-415 (1970). 
[36] J. Van-tiel, Espaces localement $K$-convexes, I-III. Proc. Kon. Ned. Akad. van Wetensch. A68, pp. 249-289 (1965).

\section{R. Ameziane Hassani}

Department of mathematics and computer science Faculty of Sciences Dhar Mahraz

B. P. 1796 Atlas Fès, Morocco

e-mail : ra.ameziane@yahoo.fr

\section{A. El Amrani}

Department of mathematics and computer science Faculty of Sciences Dhar Mahraz

B. P. 1796 Atlas Fès,

Morocco

e-mail : abdelkhalek-amrani@hotmail.com

and

\section{Babahmed}

Department of mathematics and computer science Faculty of Sciences Meknes,

Morocco

e-mail : babahmed@hotmail.com 